Anales del Seminario de Historia de la Filosofía

ISSNe: 1988-2564

http://dx.doi.org/10.5209/ASHF.56109

\title{
¿Hay nada antes del principio? Acerca del sentido y de la sostenibilidad de la idea de creatio ex nihilo en La Estrella de la Redención de F. Rosenzweig
}

\author{
Ángel Enrique Garrrido-Maturano
}

Recibido: 01/10/2015 / Aceptado: 28/03/2016

Resumen. El estudio analiza desde una perspectiva fenomenológica la idea de creación en La Estrella de la Redención de Franz Rosenzweig. El análisis determina primero en qué medida el concepto de creación puede ser considerado bajo la categoría de creatio ex nihilo y qué concretamente ha de entenderse por tal. Luego explicita hasta qué punto es legítimamente sostenible la concepción de la creación desde la nada postulada por Rosenzweig y cuál es su estatus epistemológico confrontada con un estudio científico del problema. Finalmente elucida cómo creatio ex nihilo y creatio continua se implican y presuponen mutuamente en el pensamiento rosenzweiguiano.

Palabras clave: Creación; nada; principio; revelación; Rosenzweig.

[en] Is there nothingness before the beginning? On the meaning and feasibility of the idea of creation ex nihilo in F. Rosenzweig's The Star of Redemption

\begin{abstract}
The article analyzes, from a phenomenological point of view, the idea of creation in F. Rosenzweig's The Star of Redemption. The analysis first establishes the degree in which the concept of creation can be considered under the category of creatio ex nihilo, and what is to be understood concretely as this type of creation. Then it explains the extent in which the conception of a creation out of nothingness advocated by Rosenzweig can be legitimately advanced as well as the epistemological status that can be assigned to it. Finally, the article discusses how creatio ex nihilo and creatio continua involve and presuppose themselves reciprocally in Rosenzweig's thought.
\end{abstract}

Keywords: Beginning; creation; nothingness; revelation; Rosenzweig.

Sumario. 1. Introducción. 2. Función y dimensiones del análisis de la creación en la estructura de La Estrella. 3. Lógica de la creación. 3.1. Creador y criatura. 3.2. Principio y caos. 4. 3 El concepto de creación desde la nada de La Estrella y su sostenibilidad. 5 Creatio ex nihilo, creatio continua y redención.

Cómo citar: Ángel Enrique Garrrido-Maturano (2017): “¿Hay nada antes del principio? Acerca del sentido y de la sostenibilidad de la idea de creatio ex nihilo en La Estrella de la Redención de F. Rosenzweig”, en Anales del Seminario de Historia de la Filosofia 34 (2), 433-449.

1 Doctor en filosofía; Investigador del Consejo Nacional de Investigaciones Científicas y Técnicas (CONICET); Investigador independiente; Instituto de Investigaciones Geohistóricas, Resistencia, Argentina.

hieloypuna@hotmail.com 


\section{Introducción}

El presente estudio ha de centrarse en uno de los temas que, dentro del sistema de filosofía configurado por la obra capital de Franz Rosenzweig, La Estrella de la Redención, y, particularmente, dentro del corazón de la obra, representado por el libro segundo, ha sido objeto de menor estudio y análisis específico ${ }^{2}$. Me refiero a la idea de creación.

Si bien es cierto que el libro II de La Estrella ha sido escrito "in theologos", no lo es menos que Rosenzweig declara en El nuevo pensamiento que "los problemas teológicos quieren ser traducidos en términos humanos y los humanos elevados hasta el nivel de la teología" pensamiento, igualmente afirma que este último "no se dirige únicamente a los así llamados 'problemas religiosos', (...), ni conoce aquella actitud, mezcla de ataque y defensa y nunca orientada objetivamente hacia las cosas, que es característica del pensamiento teológico"s. De acuerdo con estas declaraciones, desde el punto de vista metodológico, el tratamiento que hagamos aquí del concepto rosenzweiguiano de creación se atendrá exclusivamente al plano filosófico. Ello significa - negativamente hablando - que el análisis de la determinación, fundamentos y sostenibilidad de la noción de creación en La Estrella renuncia metodológicamente a cualquier revelación positiva y bíblica como prueba o dogma a partir del cual estudiar el fenómeno y se restringe a experiencias "objetivas", esto es, propias de todo existente en cuanto tal, con independencia de su filiación confesional. En síntesis - y para usar la expresión del propio Rosenzweig — podría decirse que el problema de la creación será tratado aquí como un problema "humano". Desde el punto de vista positivo, el

2 Una presentación general de la idea de creación en La Estrella la encontramos en el que aún hoy sigue siendo uno de los dos mejores estudios de conjunto de la obra de Rosenzweig. Me refiero a CASPER, Bernhard. Das dialogische Denken. Franz Rosenzweig. Ferdinand Ebner und Martin Buber. Freiburg/München: Alber, ${ }^{22002,}$ en especial pp. 119-124. El otro lo es, por cierto, el ya clásico de MOSĖS, Stéphane. System und Offenbarung. Die Philosophie Franz Rosenzweig. München: Wilhelm Fink Verlag, 1985, en especial pp. 76-80. La inmanencia y no la precedencia del caos al mundo, como así también el estatus no científico de la idea de creación en La Estrella, fueron explicitados por KOHR, Jörg. "Gott selbst muss das letzte Wort sprechen." Religion und politik im Denken Franz Rosenzweig. Freiburg/München: Alber, 2008, pp. 113-118. De las relaciones entre la narración de la creación en Gen. I y el concepto filosófico de creación de La Estrella se ha ocupado con detenimiento ASKANI, Hans-Christoph. "Schöpfung der Welt und Grammatik der Sprache". En: BRASSER, M. (ed.). Rosenzweig al Leser. Kontextuelle Kommentare zum Stern der Erlösung. Tübingen: Niemayer, 2004, pp. 412-428. El propio ASKANI, Hans-Christoph, en su libro Schöpfung als Bekenntnis. Tübingen: Mohr Siebeck, 2006, pp. 117-128, desde una perspectiva más cercana a la teología, ha profundizado en el análisis de la relación esencial entre creación y lenguaje. De la creación como acontecimiento temporal se ha ocupado WIEHL, Reiner. "Zeit und Zeiterfahrung im 'neuen' Denken von Franz Rosenzweig”. En: SCHMIED-KOWARZIK, W. (ed.). Franz Rosenzweigs “neues Denken” — Band I: Selbstbegrenzendes Denken: in Philosophos, Freiburg/ München: 2006, pp.299-311; especialmente pp. 309-311. Finalmente un vistazo panorámico de los análisis significativos para la comprensión del concepto de creación en la obra de Rosenzweig debetener en cuenta otros dos trabajos de los ya destacados Casper y Mosès. Me refiero respectivamente a CASPER, Bernhard. "Alles fängt und kommt aus dem Nichts." En: BRASSER, M. (ed.). Rosenzweig Jahrbuch 1. Franz Rosenzweig heute, Freiburg/Alber: 2006, pp. 97-113, que aborda la cuestión de los múltiples sentidos de la nada en La Estrella y su función en la concepción de la creación; y a MOSĖS, Stéphane. "Le dernier Journal de F. Rosenzweig." En: MONGIN, O./ ROLLAND, J./ DERCZANSKI, A. (eds.) Franz Rosenzweig, Les Cahiers de La nuit surveillée n. 1. París: La nuit surveillée, 1982, pp. 207-219; en especial pp. 217-219. Este último texto resulta esencial para comprender en qué medida el mundo creado no puede ser reducido a una mera representación de la voluntad.

ROSENZWEIG, Franz. El nuevo pensamiento. Buenos Aires: Adriana Hidalgo, 2005, p. 37. Sigla: NP.

$N P, 36$.

$N P, 38$. 
análisis filosófico de la idea de creación puede determinarse con los adjetivos fenomenológico, hermenéutico y trascendental. Fenomenológico en la medida en que, como veremos, para Rosenzweig el acontecimiento de la creación se muestra o pone a la luz originariamente a través de la experiencia humana del estar-ahí-ya-siempredado del mundo como tal, esto es, a través de la experiencia, que se plasma en el discurso narrativo, del darse de lo que se da, tal como lo dado efectivamente se da. Hermenéutico en cuanto aquello que se da requiere, por su propio modo de darse, de interpretación, esto es, de la elucidación de algo — lo que se da - como algo, para que el darse traiga o ponga a la luz su sentido creational. Finalmente es trascendental en cuanto la interpretación del darse del mundo bajo la categoría de la creación, si bien no prueba empíricamente la creación, es, en el contexto del pensamiento rosenzweiguiano, condición de posibilidad presupuesta por toda comprensión evolutiva del mundo.

Dentro de este marco metodológico nuestra reconstrucción del concepto de creación en La Estrella no pretende en modo alguno ser exhaustiva ni resumir las distintas dimensiones en las que Rosenzweig trata la cuestión (de hecho apenas nos referiremos aquí a la gramática del logos y al análisis gramatical de Gen 1 y dejaremos de lado por completo la cuestión del Islam y de la estética de la creación), sino que dicha reconstrucción y análisis crítico estará al servicio de tres objetivos específicos y correlacionados. En primer lugar, determinar en qué medida el concepto rosenzweiguiano de creación puede ser considerado bajo la categoría de creatio ex nihilo y qué es lo que concretamente ha de entenderse por tal. En segundo lugar, mostrar hasta qué punto es hoy día objetiva y no sólo teológicamente sostenible la idea de creación postulada por Rosenzweig y precisar cuál es su estatus por medio de una confrontación con un estudio científico-cosmológico del problema de la creación. Finalmente, explicitar cómo creatio continua y creatio ex nihilo se presuponen mutuamente en el pensamiento de Rosenzweig, al punto de que cada una es impensable sin la otra y las dos se funden en el mismo proceso creacional. Los tres objetivos señalados resultan de tres hipótesis específicas sobre cuya base ha de interpretarse y reconstruirse la noción de creación en la obra capital del filósofo de Kassel. Según la primera hipótesis no ha de comprenderse la creatio ex nihilo de acuerdo con el paradigma tradicional que supone un Dios "anterior" al mundo que desde la nada vacía hace ser en algún instante al mundo; antes bien: el mundo es creado desde la "nada de mundo", no desde la nada vacía, esto es, en el principio absoluto, antes del cual no tiene sentido hablar de determinación temporal alguna ni, por tanto, afirmar la existencia de un Dios que precede al mundo. El mundo recibe su existencia como mundo desde fuera de sí, es decir, el hecho de que haya meros procesos materiales mecánicos regidos por las leyes naturales y que ellos se configuren como totalidad ordenada de particulares tiene su origen fuera de dichos procesos materiales. La segunda hipótesis hace propia aquella idea de Rosenzweig, expuesta claramente en el pequeño texto "Glauben und Wissen", según la cual el pensamiento de la evolución paulatina y de la transformación constante no aclara la cuestión del comienzo, sino que "la deja irresuelta como el único enigma del mundo"; y aquella otra según la cual el relato bíblico de la creación posee un derecho frente a toda explicación natu-

6 ROSENZWEIG, Franz. “Glauben und Wissen.” En: Id.. Franz Rosenzweig. Der Mensch und sein Werk. Gesammelte Schriften III. Herausgegeben von Reinhold und Annemarie Mayer, Dordrecht/Boston/Lancaster: Martinus Nijhoff, 1985, pp. 581-596, aquí p. 585. Sigla: GS III. 
ral, en tanto en cuanto, en relación con el mundo y las leyes que lo rigen y explican, hay algo inexplicable y milagroso, a saber, precisamente, el comienzo ${ }^{7}$. Finalmente, la tercera afirma que la creación continua es ininteligible sin un comienzo y el comienzo es ininteligible como tal sin un proceso del cual es precisamente comienzo.

Para acceder a los objetivos mencionados comenzaremos situando de modo propedéutico y panorámico la función de la creación dentro de la estructura de La Estrella.

\section{Función y dimensiones del análisis de la creación en la estructura de $L a$ Estrella}

La función de la creación en La Estrella depende directamente del lugar en el que ella aparece en el entramado sistemático de la obra. En este sentido la idea de creación se halla determinada doblemente. De una parte, por el análisis de los elementos del antemundo, que la preceden lógicamente, de otra, por la revelación que la sucede temporalmente y a la cual ella misma pertenece como revelación primera u originaria. En tanto precedida por el análisis del antemundo, la creación es la instancia en que los elementos Dios, mundo y hombre dejan de ser lo que ellos eran en este antemundo, a saber, tres totalidades conceptuales atemporales y cerradas en sí, que, como tales, no se relacionan mutuamente y cuya realidad efectiva es incierta, para comenzar a ser realidades efectivamente acaecientes, que, en su acaecer, se relacionan por sí mismas unas con otras y cuyas interrelaciones (que comienzan precisamente con la creación) constituyen el propio acontecer del tiempo. La creación es así la instancia en la que el acaecimiento de Dios, mundo y hombre pone un punto final a la pura especulación, por la cual el pensamiento intenta de múltiples maneras dar cuenta por sí mismo de aquello que él por sí mismo no puede hacer ser, sino sólo experimentar y narrar: el acaecer mismo, la realidad efectiva de todo lo que es. En tanto sucedida temporalmente por la revelación de Dios al hombre (la revelación en sentido estricto), la creación, como instancia originaria o primera, forma parte de ese proceso histórico que la revelación en sentido amplio propiamente es. Rosenzweig puede por ello mismo situar la creación en el primer libro de la segunda parte, dedicada a la revelación, y afirmar que con ella "se rompe la corteza del misterio",

7 "La verdad es que aquí hay algo inexplicable. No algo incognoscible, pero sí algo irreductible. Un comienzo. Dónde reside él e incluso cuándo son cuestiones secundarias. Tiempo hubo suficiente y números también." (GS III, 584).

8 Por una parte es sin duda cierto que el tema de la creación es complejo y específico y también lo es que su contextualización dentro de la arquitectura de La Estrella, abordada en el próximo parágrafo, puede resultar insuficiente como introducción al lector no familiarizado con el pensamiento rosenzweiguiano. Por otra parte carezco aquí de espacio para una introducción a La Estrella de la Redención que no sólo se contente con vagas generalidades, sino que pudiera ser en verdad útil al lector primerizo. Por ello mismo, para una presentación general del sentido de dicha obra y de sus temáticas principales remito, ante todo, al que, a mi modo de ver, es el mejor estudio de conjunto de la obra de Rosenzweig. Me refiero al libro, arriba citado, de Stephane Mosès, System und Offenbarung. Die Philosophie Franz Rosenzweig. Otra excelente introducción al libro capital de Rosenzweig es el de GÖRTZ, Heinz-Jürgen. Franz Rosenzweigs neues Denken. Eine Einführung aus der Perspektive christlicher Theologie. Würzburg: Echter, 1992. En español me permito remitir a mi libro: La estrella de la esperanza. Introducción a La Estrella de la Redención de Franz Rosenzweig desde una perspectiva fenomenológica, Buenos Aires: Academia Nacional de Ciencias de Bs. As,, 2000, en especial el capítulo II "Del concepto mudo a la realidad coral".

9 ROSENZWEIG, Franz. La Estrella de la Redención. Salamanca: Sígueme, 1987, p. 155. En adelante: ER. 
pues "en el crear de Dios, en tanto que principio de su exteriorizarse, se exterioriza el poder divino"10; es decir, Dios se revela o exterioriza inicialmente en el mundo como Poder Creador y el mundo, por su parte, como creatura efectivamente existente. La creación, que se continúa en la revelación en sentido estricto y se consuma en la redención, es, así, el origen de toda revelación, toda vez que ésta, en tanto que relación entre Dios y hombre, supone el ser ya ahí, el estar ya creados del hombre y, por ende, del mundo en el que éste es ${ }^{11}$. En tanto origen del efectivo acaecer de Dios, mundo y hombre en su relación recíproca, no constituye un momento del tiempo, sino precisamente el origen mismo del acaecer del tiempo como tiempo que sucede y como aquello desde lo cual todo lo que efectivamente se da llega al ser, transcurre, se consuma, muere y se renueva. En esa misma medida, puede ser determinada por Rosenzweig como "el fundamento perpetuo de las cosas" 12 . En tanto inserta en el marco de la revelación en sentido amplio, la creación no es otra cosa que la efectiva y originaria revelación de la relación primordial entre Dios y mundo. La relación del mundo con Dios es su creación. Dicha relación co-implica, además, al hombre mismo, pues, en primer lugar, la creación del mundo por Dios es el fundamento del hombre, en tanto en cuanto el mundo está ya siempre ahí dado de antemano haciendo posible su existencia, que es existencia en el mundo. Pero, además y esencialmente, el hombre se haya co-implicado en la creación, en cuanto su relación con la creación radica en ser aquel ente del mundo creado que pone de manifiesto la creación como tal a través de su propio lenguaje, en cuyas categorías gramaticales se testimonia el proceso creativo de objetivación del mundo. El hombre es aquel que, narrando el llegar a darse de lo que se da, deja que se diga la creación. En consecuencia, si la creación es la revelación originaria de la relación entre Dios y mundo, la revelación de la creación como tal se cumple a través de la narración del hombre del estar ya siempre ahí del mundo.

De acuerdo con esta doble determinación de la creación, su función no consiste meramente en ser lo que sucede al comienzo. Mucho más importante es el hecho de que con la creación el fin del antemundo revierte en el comienzo del mundo real y efectivo $\mathrm{y}$, consecuentemente, en el comienzo de la efectividad y vitalidad del Dios viviente. Este comienzo de la creación no es un inicio lejanísimo y perdido en un pasado remoto, sino que lo ya creado está una y otra vez revelando ahora su creaturalidad, en la medida en que el lenguaje narra la creación y, así, hace presente el proceso por el cual lo creado (el mundo objetivo) ha llegado a darse tal cual se da. En el libro primero de la segunda parte de su obra capital Rosenzweig analiza esta función de la creación desde tres dimensiones diferentes. En primer lugar desarrolla sistemáticamente la relación a través de la cual Dios se revela como fundamento creador del mundo y el mundo como criatura fundada en Dios, es decir, pone a la luz lo que la creación significa tanto para el creador como para la creatura a través de un

$10 \quad E R, 156$.

11 La continuidad procesual entre creación que es en sí misma revelación y revelación que se concreta como creación ha sido descrita por R. Wiehl en los siguientes términos: "La creación de Dios es creación a la luz de la revelación. La revelación se realiza de cara al mundo creado en la creación del hombre.” (R. Wiehl, op. cit., p. 309). A mi modo de ver, Wiehl enfatiza aquí el hecho de que la revelación en sentido estricto, que acontece entre el hombre, como sí mismo irreductible al mundo físico, y Dios, presupone la creación del hombre como "personalidad", es decir, como parte del mundo. Dicho de otro modo: el aspecto propiamente espiritual del hombre, presupone su realidad como ente físico del mundo. Por ello mismo la creación ocurre entre Dios y el mundo.

$12 \quad E R, 155$. 
análisis de la lógica de la creación. En segundo lugar, desarrolla una reflexión acerca de la posibilidad de conocer por medio de las estructuras esenciales del lenguaje el proceso creacional que lleva a la objetivación del mundo. Se plantea, así, la pregunta de en qué medida y bajo qué categorías el lenguaje puede ser el "organon" en el que y a través del cual la creación se revela y se nos da. Estamos en el ámbito de la gramática del logos. Finalmente, Rosenzweig cierra el libro primero de la segunda parte con un análisis gramatical del relato bíblico de Gen. I. Este análisis resume y presenta en clave teológica lo que de modo filosófico ya había sido dicho en la lógica de la creación y en la gramática del logos. De este modo se concreta la intención, anunciada en El nuevo pensamiento, de traducir los temas teológicos en términos humanos y, a la vez, de elevar los temas humanos al nivel teológico. El análisis gramatical de Gen. I muestra cómo lo analizado filosóficamente acerca del concepto de creación converge o, por así decir, se refleja o testimonia en otro lenguaje y desde otra fuente: la de la revelación bíblica positiva, pero sin que el análisis de la narración del texto bíblico venga a cumplir la función de fundamentar o probar el análisis filosófico antes desarrollado ${ }^{13}$.

Es precisamente la primera de las tres perspectivas o dimensiones señaladas, a saber, la del estudio de Dios como creador, del mundo como creatura y de la relación lógica entre ambas instancias, aquella en que se pone de manifiesto filosóficamente el sentido y la sostenibilidad de la concepción rosenzweiguiana de la creación ex nihilo, que luego habrá de mostrarse como tal en la estructura gramatical del lenguaje y, además, ser narrada en el texto bíblico. Es, por tanto, esta primera perspectiva, la que concentrará nuestra atención.

\section{Lógica de la creación}

\section{1. Creador y criatura}

Para Rosenzweig, Dios, si no quiere ser confundido con o reducido a algo distinto de sí - el hombre o el mundo_-, sólo puede ser pensado de acuerdo con su concepto lógico específico, tal cual este concepto es construido metódicamente en Estrella I, a saber: como un ser que afirma (sí) su ser o "así" como un puro ser infinito, una infinita facticidad ${ }^{14}$, y que niega (no) su nada y pone el ser infinito que él es a través de un acto infinito e incondicionado de libertad. Una libertad que pone un ser infinito es una libertad infinita; y una libertad infinita es una libertad que se puede identificar con el poder infinito. Consecuentemente, el concepto de Dios sólo puede ser configurado por el pensamiento como el de una vitalidad que tiene el poder de convertir su libertad en esencia. Entonces, si Dios efectivamente existe, en su revelación en

13 En este punto afirma con razón Hans-Christoph Askani que en lo que respecta al estudio de Gen. 1 "no se trata de una interpretación o exégesis en el sentido clásico, sino de un 'análisis gramatical', una consideración del texto que encuentra y extrae su propio mensaje de la percepción de los propios elementos y operaciones lingüísticas del texto; como si se hubiera depositado en el lenguaje, hasta en su estructura y hasta en el interior de sus formas, el contenido y mensaje del propio texto." (H-C. Askani, Schöpfung als Bekenntnis, p. 119). En el mismo sentido, en otro texto suyo, afirma: "La Biblia no es la fuente, sino el testimonio de la revelación. (...). La Biblia simplemente narra aquel acontecimiento del cual la 'gramática del logos' es su recepción, su reconocimiento filosófico, a saber: nos habla del lenguaje no como mero instrumento o soporte de la comprensión, sino como don de la revelación." (H-C. Askani, "Schöpfung der Welt und Grammatik der Sprache”, p. 424).

14 Cfr. $E R, 68$. 
la realidad efectiva deben reconocerse las determinaciones incluidas en su concepto, pues, en caso contrario, lo que se revelaría no sería Dios. Ahora bien, en la realidad efectiva, el "no" por el cual el concepto de Dios niega su nada — su poder infinitosurge, ya no como "no", no como acto repentino de negación, sino como "sí", como afirmación constante de una propiedad infinita y duradera; y lo hace precisamente en el poder creador, que se extiende siempre y por doquier, y que se manifiesta en el haber podido llegar al ser de todo lo que es. Pero: “¿qué es el poder, una vez que ha llegado a ser propiedad?" 15 Pues no otra cosa que la negación misma de la nada de Dios y la afirmación de su esencia. Dios, si efectivamente existe, no puede ser sino infinito poder creador, porque sólo como Poder Creador, que se da desde siempre y en todo lo que es, puede manifestarse la esencia infinita de Dios. Por ello puede afirmar Rosenzweig que "el Dios visible de la creación, puede cuanto quiere, pero sólo quiere lo que por su esencia tiene que querer" ${ }^{\prime \prime}$. A Dios, en virtud de su propio concepto, para ser efectivo y manifestar su poder infinito, le es una necesidad esencial crear el mundo; y sólo con la creación satisface la necesidad de su naturaleza. Sin embargo, esta necesidad esencial no constituye ninguna menesterosidad por la que Dios estuviera condicionado y que lo pudiera privar de su libertad infinita, pues, aunque bien es cierto que Dios, en cuanto decide revelarse, no puede sino crear ${ }^{17}$, el que Dios mismo se revele resulta del "el núcleo íntimo de libertad sin límites"18 que define el concepto de Dios. Esta convergencia de arbitrariedad y necesidad en el acto creador nos permite destacar dos aspectos decisivos para comprender en qué medida la creación es creación desde la nada. Primero, que no es fácticamente posible la revelación, esto es, el darse efectivamente de Dios sin la creación, y que, por tanto, es impensable un Dios viviente subsistiendo no se sabe muy bien cómo en la nada. Y, segundo, que lo que antecede a la creación no es la nada vacía, un limbo tenebroso y atemporal en el que Dios flotaría, sino "la autorrevelación precreacional de la libertad divina" 19 . Con dicha autorrevelación mienta Rosenzweig el hecho de que Dios en cuanto tal experimenta y no puede no experimentar que Él tiene infinita libertad para crear y que - justamente porque el poder de crear es un poder de su libertad - nada lo obliga a ello. Por ello mismo, que efectivamente cree es experimentado por Dios como una arbitrariedad. Dios, para ser efectivamente Dios, tiene necesariamente que crear, y una nada anterior a la creación, de la que ésta hubiera surgido, es incompatible con un Dios efectivo y realmente existente; es una mera construcción conceptual. Pero que haya Dios y que efectivamente cree, esto se funda en nada, porque el ejercicio, por parte de Dios, de su propia creación es el principio arbitrario del ser. De ahí que, desde la perspectiva del creador, la creación sea una creación desde la nada. Sin embargo, el principio no es la nada vacía, ni Dios en la nada, ni un Dios que existiera antes de la creación y creara al mundo desde la nada absoluta. "El principio es: Dios creó20".

Y Dios creó el mundo. Sólo el pensamiento de la creación arranca al mundo de su condición de elemento, de ser la mera idea de un todo ordenado de particulares configurados lógicamente según géneros, especies e individuos, para lanzarlo a la

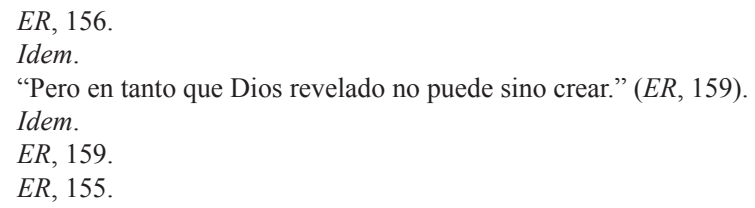


corriente del ser. Sin embargo, ¿no resulta paradójico sostener una creaturalidad del mundo posterior a su acabamiento como idea? Se pregunta Rosenzweig: “¿Es que acaso tendría que volver a convertirse en nada el propio mundo configurado, para representar la 'nada' a partir de la cual se cree el mundo"21 Y su respuesta es contundente: "Así es, en efecto"22. El mundo es creado desde la nada, no necesariamente porque a él le hubiera precedido el silencio de los infinitos espacios vacíos, ni porque su propia materialidad y las formas o leyes de acuerdo con las cuales se va configurando como mundo no hayan estado dadas desde el principio, sino porque el principio es la creación y la creación es ya creación del devenir del mundo. El mundo es creado desde la nada porque la existencia del mundo es autónoma respecto de la idea del mundo; porque, contra toda presuposición idealista, nada en su idea, nada en la totalidad orgánica y racional de la idea del mundo, le puede dar a este mundo consistencia y facticidad. Esta le viene dada, por así decir, desde la nada de sí mismo, desde la nada de mundo. Por ello mismo, desde la perspectiva de la creatura, la creación es creación desde la nada. En efecto, cuando el nuevo pensamiento afirma, como lo hace, "la autonomía del mundo, su precedencia a todo pensamiento y la cualidad de su existencia, en ello está implicado que el mundo ha sido creado desde la nada"23. Su creación desde la nada no implica, pues, el haberse creado de una vez y para siempre desde el vacío anterior al principio, porque el principio es la creación y lo anterior al principio es un absurdo. Su creación desde la nada implica, antes bien, "la irrupción de la conciencia de su creaturalidad"24. Tal irrupción no puede significar, a mi modo de ver, sino el hecho de que el mundo no se manifiesta como habiendo sido creado de una vez y para siempre desde el vacío, sino como aquello que, desde fuera de sí, desde la nada de sí, está desde siempre siendo creado, como aquello que está siendo "perpetuamente criatura" 25 . En términos del propio autor: "La relación que buscamos entre el mundo y el creador era para el mundo, (...), no su estar de una vez por todas y para siempre creado, sino su reiterado revelarse como criatura" 26 . En efecto, la existencia de todo lo particular configurado como particular de un género en virtud de un orden jerárquico de universales, exige, precisamente por la constante instantaneidad e incesante renovación de la existencia, que una y otra vez le sea suministrado el poder de llegar a ser y configurarse; exige, entonces, estar siendo constantemente creada y recreada. "Y es así como la toma en su mano el poder del Creador"27, quien, habiéndola creado desde la nada, la recrea constantemente.

\subsection{Principio y caos}

Es cierto: la existencia exige ser constantemente recreada por el Creador. Pero, ¿cuándo fue creada por vez primera? ¿Cuál es el principio de la creación? En el contexto del análisis de La Estrella y del entero nuevo pensamiento, la pregunta no tiene sentido. En primer lugar, debemos tener en cuenta el principio fundamental del

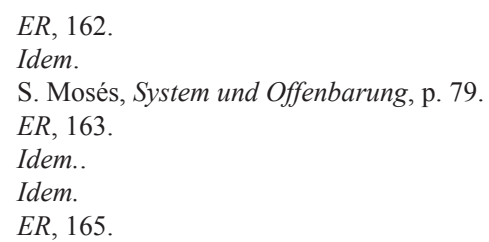


nuevo pensamiento, a saber, que los elementos y el tiempo mismo sólo existen y comienzan propiamente a ser reales (y no meramente conceptos lógicos) en cuanto se revelan y relacionan en la realidad efectiva ${ }^{28}$. En segundo lugar, debemos también tener en cuenta que la relación primigenia es la creación del mundo por Dios, que se le revela al hombre en las categorías gramaticales ${ }^{29}$ de su propio lenguaje narrativo. $\mathrm{Si}$ consideramos estos dos aspectos, entonces tenemos que comprender que no hay un principio de la creación y, consecuentemente, un "antes" verdaderamente temporal y no lógico en el que ésta hubiera ocurrido, sino que la creación es el principio, lo iniciático, lo que está "antes de todo". Escribe Rosenzweig: "El mundo existe antes de todo, aprendemos ahora. Existe simplemente. Este ser del mundo es su Existir-ya, su Estar-ya-ahí. ‘¿Qué haces en el mundo? El mundo está ya hecho." 30 El 'estar-ya ahí del mundo', el hecho de que el mundo nos está dado antes de que hagamos cualquier cosa y antes de cualquier acontecimiento, que es siempre acontecimiento del mundo, lo conocemos como la nota decisiva de la creación, en el doble sentido de que pone de manifiesto que el mundo ya ha sido hecho, de que su creación nos antecede y hace posible la existencia de todo particular; y de que la creación, en tanto presuposición originaria, es el comienzo mismo antes del cual todo ahí espacial o ahora temporal es impensable. En la experiencia del estar-ya-ahí del mundo, que todo hombre y todo ente presuponen en cuanto comienzan consigo mismos, confluyen tanto la creaturalidad del mundo como el poder creador de Dios. En efecto, el mundo real está ya ahí siempre dado como una totalidad que a cada instante acontece de modo diverso, conteniendo dentro de sí lo particular y ordenándolo de acuerdo con leyes universales. Este mundo, para ser real, para estar ahí dándose con su constante instantaneidad, necesita que su existencia esté siendo constantemente creada y renovada. Necesita, para ser real, consistencia, es decir, duración en el tiempo, y verdad, es decir, universalidad, en el sentido de acaecer conteniendo como totalidad universal la plétora fenoménica. Mas tales cosas no puede garantizárselas al mundo ni su ideal metalógica, ni los meros procesos materiales que, en cuanto son, ya existen. Su existencia, su consistencia y su verdad tiene pues que serle dada desde un ser fuera de sí, es decir, desde la nada de sí. Y en tanto ello ocurre, el mundo, en su estar ahí dándose, revela su creaturalidad inicial y, a una con ello, el poder creador que le dio su creaturalidad.

El acontecimiento de la creación aportó al mundo aquello que ni él podía darse por sí mismo ni tampoco podían aportarle las leyes que rigen cómo se da el mundo: su existencia, su "estar-ya-ahí", que es aquello primero, primi-genio y "ya puesto", que presuponen los mismos conceptos científicos que intentan explicar el mundo, en tanto en cuanto ellos se remiten y se aplican a un pasado ya dado que intentan determinar. Al darle al mundo su "estar-ya-ahí", la idea de la creación da también el tiempo mismo, hace ser el "ya" primero, el acontecimiento primigenio con el cual se inaugura el tiempo, pues, para Rosenzweig, el tiempo no es una magnitud abstracta, no es el tiempo "en el que" algo acontece, sino el tiempo que se despliega como

28 En este sentido y con admirable precisión afirma Hans-Christoph Askani: "En la creación, Dios deviene creador, esto no es sólo para el mundo, esto es también un principio para Dios. Ahora Él ya no es sólo Dios, sino el Dios real: Dios el creador del mundo". (Schöpfung als Bekenntnis, p. 120).

29 No podemos aquí entrar en esta cuestión que exigiría por sí misma un estudio específico. Para un análisis detallado de la función de las distintas categorías gramaticales en el análisis rosenzweiguiano de la creación cfr. ASKANI, Hans-Christoph. "Schöpfung als Sprachgeschehen in Rosenzweigs 'Stern der Erlösung"”. En: id., op. cit., 117-128; particularmente pp. 121-125.

$30 \quad E R, 175$. 
acontecer de lo que acontece, esto es, el "tiempo que acontece por sí mismo"31. E igualmente, dándole al mundo su "estar-ya-ahi", la idea de la creación da el espacio mismo, el "ahí" en el cual puede haber espacio, puesto que todo ahí se remite a un algo o, para usar la expresión de Rosenzweig, a un "Este", a alguna cosa cualquiera de la plétora del mundo, que es "ahí". Por eso "el mundo no es originalmente espacio" 32 , sino que el espacio es originalmente mundo. "Al aquí lo precede el Este" y el Este como condición del aquí precede al espacio ${ }^{33}$. Este acaecer de la plétora del "Este" o de los "algos" que, en tanto acaecer primero, inaugura el tiempo, y que, en tanto posibilita un "ahí", hace lo propio con el espacio, constituyen "las primicias de la Creación" 34 , aquello a partir de lo cual se renueva y temporaliza el tiempo y se espacia y reconfigura el espacio. Este acaecer, que en última instancia es el acaecer de la creación, constituye, así, el "desde dónde" o principio del que mana toda plenitud o plétora y gracias a la cual acontecen el espacio y el tiempo, pues ellos sólo son pensables como el espacio que se dibuja entre lo que acaece y como el tiempo que emerge con el acaecer mismo de lo acaeciente. La existencia, que es lo que el acontecimiento de la creación da al mundo, permanece, entonces, como "el fundamento inmediatamente creado, el principio del que manan los partos siempre nuevos de la plenitud" 35 . No es necesario, como en el idealismo, presuponer un caos anterior a la generación del mundo para explicar la materia de la que se genera o emana el mundo, que luego será constituido como un todo orgánico por el sujeto o espíritu puro. El caos no es una singularidad presupuesta por la creación, sino que la creación —origen de todo espacio y de todo tiempo- crea la existencia misma del caos. "El caos está en la Creación, no antes de la Creación. El principio está en el principio"36. Y si el caos originario, incluso aquel que presuponen las cosmologías actuales, está en la creación, ello significa que la creaturalidad del mundo es tanto finita, en cuanto no es su propio origen, cuanto necesitada de consumación. "El mundo como creación no es el cosmos ordenado. En tanto lo general que está lleno de lo particular, el mundo debe aún ser ordenado" "37. Por ello mismo el caos es en él y no antes de él y por ello mismo también la creación continúa.

La idea de creación, tal cual Rosenzweig la entiende, es decir, como creación incluso del caos (concebido como una instancia germinal en el proceso del irse desarrollando del mundo) y no como creación desde el caos, "manifiesta qué importancia real tiene el concepto de creación de la nada (...)"38, pues ella es la única manera de no presuponer arbitrariamente el caos y, entonces, enfrentarse o bien al problema de cómo del caos habría surgido el logos - el conjunto de leyes universales - que lo ordena, o bien al de cómo del logos universal, que es siempre universal respecto de los particulares de los cuales se enuncia, hubieran surgido estos particulares ${ }^{39}$. Ahora bien, si contraponemos la idea de creación, como principio absoluto al que no ante-

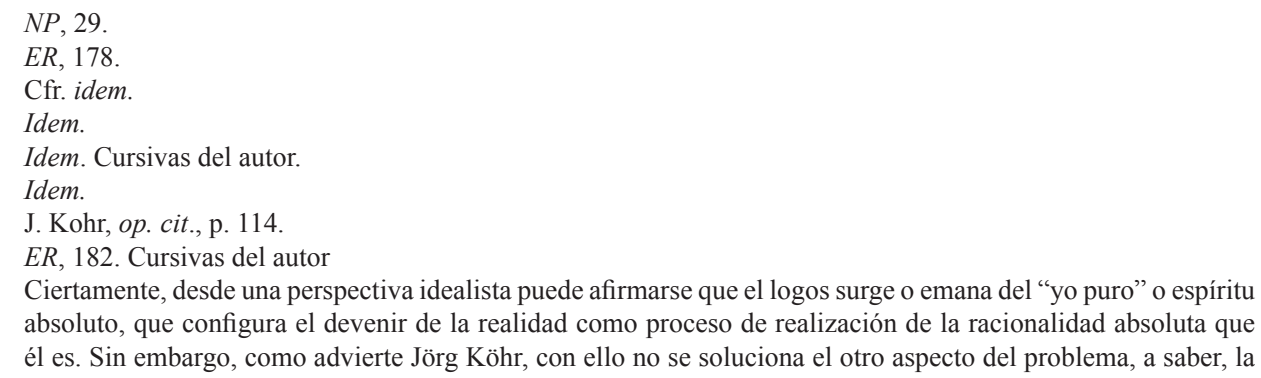
absoluto, que configura el devenir de la realidad como proceso de realización de la racionalidad absoluta que él es. Sin embargo, como advierte Jörg Köhr, con ello no se soluciona el otro aspecto del problema, a saber, la 
cede nada, a esta idea de la emanación del mundo desde el caos, se advierte que el Poder Creador $d a$ o hace ser tanto a los particulares enunciados en el mundo, como a los universales que los enuncian como tales particulares a lo largo de la historia de la creación (que, para Rosenzweig, concluye con la redención). En este sentido afirma el filósofo que "la plenitud caótica de lo particular es las primicias de la Creación, mientras que lo universal es los vasos dados, puestos por el Creador, en los que se va trasegando lo particular, que hierve y desborda libremente en la Creación" ${ }^{40}$. Así, pues, el concepto de creación desde la nada, en tanto evita la presuposición de un caos originario y los problemas que ésta suscita, "estaría perfectamente en su sitio en una confrontación entre conceptos del mundo"41. Pero, ¿lo está verdaderamente? ¿Hasta dónde es sostenible la creación desde la nada? ¿Hasta dónde la experiencia del "estar-ya — ahí-dado" del mundo es una experiencia de la creación?

\section{El concepto de creación desde la nada de La Estrella y su sostenibilidad}

Los desarrollos precedentes nos permiten ahora determinan tanto negativa cuanto positivamente los aspectos esenciales de la concepción rosenzweiguiana de la creación como creación desde la nada. Así, por un lado, el factum de que los elementos sólo adquieren realidad efectiva en el acaecimiento de su relación mutua, el cual constituye un principio fundamental del nuevo pensamiento, y, por otro, la idea de la creación como principio absoluto u origen, que no reconoce antecedente real-efectivo de ninguna clase en cuanto "el principio está en el principio", impiden, desde el punto de vista negativo, aplicar a la noción rosenzweiguiana tanto la comprensión tradicional de la creación, cuanto la lógica idealista de la emanación. En efecto, la idea de un Dios anterior a la creación, que subsiste en la vacuidad y, desde esa nada, crea el mundo en un instante es inconcebible. En primer lugar, como vimos, porque no tiene sentido hablar de anterioridad, que es una determinación temporal, respecto del principio del tiempo que es la propia creación; y, en segundo lugar, porque, si bien es un factum inexplicable y en este sentido arbitrario el que haya un Poder que cree el mundo, sin embargo, el Poder Creador (que es lo que debe comprenderse aquí como Dios desde una perspectiva fenomenológica), en cuanto es, sólo puede crear. Por lo tanto, aunque el Poder Creador es el origen, Él, el mundo y el tiempo son coetáneos: la creación que los vincula es el acaecimiento primero con el que comienza el tiempo en tanto que tiempo que acontece por sí mismo, no en tanto que ficción lógica de un tiempo abstracto y vacío "en" el que algo (o nada) acontece.

La idea de la creación rosenzweiguiana se opone también, según dijimos, a la precedencia de una suerte de caos material originario del que habría emanado el mundo. Si todo, como las cosmologías actuales parecen defender, comenzó por el caos, por una singularidad material caótica, entonces ese caos no precede al principio, sino que es el propio principio de la creación, que es tan inmemorialmente antigua como el mismísimo caos. Él es sólo el momento primero, la efectividad originaria de un Poder Creador, que sigue actuando en el mundo en su perpetua renovación y cuya

necesidad de que lo absoluto, que debería carecer de todo presupuesto irracional, tenga que presuponer el caos, porque "las formas lógicas surgen del yo, no así la materia." (J. Kohr, op. cit., p. 116).

$40 \quad$ ER, 183.

41 Loc. cit. 
obra continua, que no ha acabado hoy ni tiene por qué detenerse mañana, inaugura cada día el futuro.

La propia determinación negativa de la comprensión de la creación desde la nada nos pone de cara a su determinación positiva. Ella debe, ante todo, tener constantemente presente que, como afirma B. Casper, para el nuevo pensamiento "la existencia ya no resulta más comprensible por sí misma" y que, por tanto, "ella aparece necesitada de otro" 42 . Eso Otro real, pero que no es mundo y que hace que el mundo efectivamente exista, es el Poder Creador que llamamos Dios y cuya obra, la existencia, es la creación. Una creación que no sólo ocurre de una vez y para siempre, sino que constantemente continúa, puesto que "el estar ahí de la existencia resulta una y otra vez necesitada de una constante renovación de su estar ahí"43. El mundo per-durable, que "está-ahí" en la realidad, no funda su "estar-ahí" ni en su propio concepto, que pertenece a la irrealidad del antemundo, ni en sus leyes, que lo presuponen en tanto a él se aplican, sino que encuentra que su "estar-ahí-existiendo" le está ya dado. Por lo tanto, aquello "otro", que presta al mundo la consistencia y verdad que él ansía, tiene que ser "un ser que esté fuera del mundo, pero en el circuito de la realidad" 44 . Debería tratarse, pues, de un ser que, desde la perspectiva del mundo, sea nada de mundo. He aquí el sentido propio de la creación desde la nada. En primer lugar, el mundo es creado desde la nada porque es creado desde fuera de sí mismo, desde lo que, para el mundo, es nada; y, en segundo lugar, lo es porque el mundo es creado sin que hubiera nada que determinara necesariamente que el mundo debería ser. La creación del mundo no tiene fundamento porque la existencia del mundo y de las leyes que lo configuran es el fundamento último de todo lo dado. E1 mundo es creado desde fuera de sí sin un fundamento necesario, y en este preciso sentido, el mundo es creado desde la nada. Ahora bien, indiscerniblemente de esa existencia a la que se halla arrojado y como núcleo íntimo de su existir, ha sido dado al mundo y a su materialidad originaria su "logos", su principio ordenador, en última instancia, las leyes naturales. Ellas sí explican por qué la materialidad se desarrolla tal cual lo hace y por qué sucede todo lo que sucede tal cual sucede, sin explicar, por cierto, por qué ellas mismas suceden. Estas leyes dan al mundo su propia necesidad y sus propias verdades, sin que el hecho de que haya verdad y que el mundo tenga su propia necesidad se funden en el mundo mismo. Por ello precisamente puede decirse que "tanto el siempre renovado tener la posibilidad de ser, como la necesidad y verdad le son otorgadas al mundo desde la nada de sí mismo"45. El mundo se revela, así, concomitantemente y sin que ello implique contradicción alguna, como criatura, a la que le ha sido dada desde la nada de sí su haber llegado a estar ahí existiendo efectivamente, y como objetividad, que evoluciona y se configura por sí misma de acuerdo con sus propias leyes. "Su creaturalidad no va en absoluto en desmedro de su mundanidad"46.

Ahora bien, sostener que el mundo se revela como habiendo recibido la existencia desde la nada de sí y sin que haya nada en sí que fundamente racional y necesariamente su existencia, sin que, en una palabra, se pueda decir con razón suficiente por qué hay ser y no nada, equivale a decir que la existencia del mundo es un milagro. La

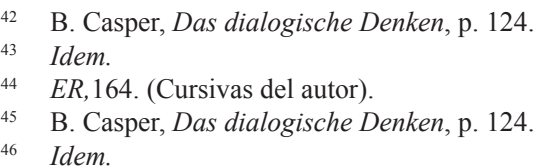


creación desde la nada en Rosenzweig es sinónima de la creación como milagro. Por eso mismo, con el tratamiento de la idea de creación estamos, dentro de la estructura de La Estrella, en "el paso" del misterio (de la imposibilidad de determinar exclusivamente en función de su propio concepto si los elementos de Estrella I efectivamente existen o no) al milagro (de su efectiva existencia). "El revelarse del perpetuo secreto de la Creación es el milagro, siempre renovado, de la Revelación. Estamos en el paso: el paso del Misterio al Milagro"47. En efecto, la primerísima revelación de Dios es su revelación como Poder Creador a través de la creación del mundo; y la creación del mundo, que no tiene razón de ser y que no puede demostrarse, es un milagro. ¿Cómo puede sostenerse un concepto que pone en el origen del mundo un milagro?

La respuesta a la pregunta precedente exige distinguir dos planos. Primero, determinar en qué medida es legítimamente posible sostener la hipótesis de la creación; y segundo, determinar cuál es el estatus epistemológico del concepto. Respecto del primer punto, Rosenzweig observa que al concepto de creación no se opone tanto la idea de ley natural, porque, como vimos, la creaturalidad del mundo no va en desmedro de su objetividad, cuanto la idea del desarrollo (Entwicklung) paulatino y progresivo de una existencia sempiterna, lo que el autor llama "historia de la creación natural (natürliche Schöpfungsgeschichte)"48. A esta concepción se le podría oponer -más allá de Rosenzweig, pero, estimo, no de modo incompatible con su propio pensamiento - la sencilla idea de que la supuesta sempiternidad no elimina la posibilidad de una creación, sino que la modifica, convirtiéndola en creación sempiterna y suponiendo un Poder Creador igualmente sempiterno. Pero si este mismo argumento es posible, ello se debe a que, como afirma Rosenzweig, la historia natural de la creación como desarrollo contínuo, en última instancia, "no aclara el comienzo, sino que, por el contrario suscita (...) el problema del comienzo" $"$. Lo deja simplemente sin resolver y, negando el milagro, consuma el misterio.

Este concepto desarrollista cree, en última instancia, que es posible aplicar al principio aquella perspectiva de la evolución que ella emplea en el centro de la historia de la creación, con el surgimiento paulatino y progresivo de la vida y, sobre todo, del hombre. Por ello sostiene Rosenzweig que "el punto sensacional de la historia natural de la creación no reside en el comienzo, sino en el medio (...)"50. Su esencia radica en la progresividad de los nexos evolutivos. Por más que dos instancias de la creación resulten esencialmente disímiles, entre ellas hay un tránsito paulatino, lleve éste el tiempo que lleve. Aquí "paulatino" significa "natural". A esta hipotética negación del comienzo y absolutización de los tránsitos paulatinos les corresponde una negación paralela de toda teleología. En contra de una concepción tal, bien puede sostenerse que no se ve por qué, aun aceptando que en el medio de la creación se dé la progresividad, ello nos adjudicaría el derecho de transpolar esta afirmación al comienzo. Tampoco se advierte en qué medida es menos hipotético sostener la falta de todo fin, que sostener un fin hacia dónde la evolución va evolucionando. Incluso no es posible hablar de evolución o desarrollo, si, de un modo latente, no se supone un "hacia donde" teleológico del desarrollo que estuviera en germen desde el comienzo, esto es, sin suponer un

\footnotetext{
$E R, 132$.

GS III, 585 .

Idem.

Idem.
} 
principio o un fin. En el fondo, el problema que afronta esta perspectiva es la dificultad de explicar por medio de lo paulatino las diferencias esenciales: el surgimiento de la conciencia de lo no consciente, de la vida de lo no vivo, $\mathrm{y}$, ante todo, de la existencia de lo no existente. Es cierto que para que lo nuevo surja deben desarrollarse las condiciones que permitan su cumplimiento y, en este sentido, lo nuevo supone el desarrollo de lo que lo precede. Pero el desarrollo lo es siempre de algo nuevo, de lo que nace y en lo que se advierte la continuidad de la obra creadora. En este sentido, el salto, lo repentino, la inflexión en la continuidad homogénea devienen la condición a través de la cual el desarrollo es posible como tránsito de lo nuevo desde lo que surge a lo nuevo que va preparando. La dificultad de esta absolutización del pensamiento del desarrollo y de la evolución es que no puede explicar la idea misma de transformación paulatina, en la medida en la que comienzo y fin son lo que determinan el "entre" que es la transformación misma en cuanto tal. En otros términos: no puede explicar cómo a lo largo de la progresión paulatina es en absoluto posible una progresión o un desarrollo. Para hacerlo es necesario admitir un claro o un hueco en la progresividad; admitir "el carácter repentino del acontecimiento" 51 , desde el cual y hacia el cual el desarrollo se desarrolla. Este acontecimiento es precisamente la creación de la existencia de lo dado y la recreación continua de lo nuevo desde lo dado. La creación deviene, así, la condición que hace posible toda historia que quiera ser más que un caos de subsistencias dadas, que quiera ser plexo y evolución, en vez de ser la evolución el indicio de la necesidad de suprimir la idea de la creación. En síntesis, la idea de creación frente a la idea de evolución es cosmológicamente sostenible en dos sentidos fundamentales: primero, porque la evolución es posibilitada por la creación, en tanto evolución desde un comienzo que determina el final hacia el cual la evolución evoluciona; y, segundo, porque ninguna respuesta al cómo del mundo, por más detallada y racional que ésta fuese, resuelve el enigma de su existencia ni logra, por tanto, impugnar la idea de milagro como respuesta a su porqué.

En cuanto al estatus epistemológico de la sostenibilidad de la idea de creación es menester puntualizar que el intento de legitimar filosóficamente la creación desde la nada de ninguna manera significa probar la creación ni otorgarle un estatus científico, sino precisamente eso: legitimar su postulación y mostrar en qué medida su carácter milagroso no puede por completo ser dejado de lado sin hundir la cuestión del comienzo en el misterio que nada explica. Ahora bien, esto, desde la lógica interna de La Estrella, no constituye ningún inconveniente, porque la creación pertenece a la revelación y, por tanto, no es ni un argumento, ni un concepto, ni una teoría que pudieran demostrarse, sino un factum que puede mostrarse o testimoniarse; y que se muestra en el "estar-ya-siempre-ahí" del mundo y en las categorías gramaticales del lenguaje que lo narran.

Precisamente en la narración del relato bíblico del Génesis se nos revela el mundo como lo hecho, lo que "está-ya-ahí" habiendo sido creado. Ya ahí, ya dados, acabados como lo creado a lo que se refiere, están también los tres momentos fundamentales de la narración en tanto acontecimiento lingüístico, a saber, el tiempo narrado, que está ya ahí en tanto pasado relatado; el lenguaje de la narración, que está ya ahí dado como gramática; y el otro al que se narra, que está ya ahí como mero oyente ${ }^{52}$. Pero

52 Cfr. GÖRTZ, Heinz Jürgen. Franz Rosenzweigs neues Denken. Eine Einführung aus der Perspektive christlicher Theologie. Würzburg: Echter, 1992, p. 82. 
Rosenzweig es plenamente consciente de que ni su análisis de la lógica de la creación, ni el relato que relata el "estar-ya-ahí" del mundo son una prueba de la creación, pues "la creación misma no es probada por el mundo" 53 . "No es posible creer en la creación porque ofrezca una explicación suficiente del enigma del mudo"54. Y ello simplemente porque no la ofrece; porque la idea de la creación desde la nada, esto es, la idea del milagro, no constituye ni puede constituir una hipótesis científica que, como toda hipótesis científica, supone el mundo y se basa en las leyes de un mundo ya dado. Sólo es posible creer en la creación, no probarla. El análisis se limita a legitimar esa creencia, a mostrar que ella es sostenible y que al comienzo hubo, al menos, la posibilidad del milagro (como explicación del misterio). Pero la creencia, por su parte, no surge del análisis, sino de la experiencia de la revelación: "El que no ha sido alcanzado por la voz de la Revelación no tiene derecho a suponer el pensamiento de la Creación como si se tratara de una hipótesis científica" ${ }^{55}$. Dicha experiencia es la experiencia de la creación continua y de nuestra participación en ella.

\section{Creatio ex nihilo, creatio continua y redención}

Para Rosenzweig no es posible creer en la creación desde la nada — en el pasado de la revelación - sin experimentar la actualidad de la revelación, que no es otra cosa que la continuidad de la manifestación del Poder Creador en el presente viviente, pues creatio ex nihilo y creatio continua se presuponen una a otra. En efecto, no es pensable ningún proceso de renovación ni de vitalización de lo que existe, ninguna destrucción ni consumación, sin que algo efectivamente exista. Dicho brevemente: la muerte presupone el nacimiento, así como el complejo proceso de creación, que se desarrolla a lo largo del día del mundo, presupone la aurora primera de la creación inicial. Pero lo opuesto también es cierto: la creación primera no se da fácticamente como un acontecimiento puntual, que se hubiese producido de una vez y para siempre, sino que en ella el Poder Creador emerge como propiedad esencial y duradera, que se extiende a lo largo del tiempo y se manifiesta en la constante reconfiguración del cosmos. Es cierto: el mundo "está-ya-ahí", "ya hecho"; pero no lo está de una vez y para siempre; antes bien, el mundo se desarrolla en la realidad efectiva al modo de un proceso vivo, si se comprende la vida en un sentido amplio, es decir, como constante génesis, desenvolvimiento, desaparición y regeneración de estructuras materiales complejas. La naturaleza, considerada desde la perspectiva dinámica del nuevo pensamiento, que toma en serio el tiempo y la relacionalidad de los acaecimientos que hacen ser al tiempo, y no desde la perspectiva estática del substancialismo, puede ser vista como un proceso que, a través de relaciones, es capaz de regenerar múltiples formas estructurales. Si admitimos que lo que desde un principio ha recibido la capacidad de regenerarse y de generar nuevas estructuras, está, en un sentido amplio, vivo, bien podemos considerar el mundo natural como un todo viviente en proceso continuo de creación y vitalización. Este proceso es, además, posible y tiene un carácter objetivo gracias a las leyes que lo rigen. Si esto es así, la creatio ex nihilo se concreta como creatio continua. Son ésta y las leyes que la rigen las que, cada día,

\footnotetext{
$E R, 178$.

ER, 179 .

Loc. cit.
} 
brindan a aquella consistencia y verdad, y las que confieren objetividad al proceso evolutivo ${ }^{56}$.

Hemos dicho que, para Rosenzweig, el existente sólo puede creer en la creación como revelación primera si es alcanzado por una revelación ahora acaeciente ${ }^{57}$. Luego afirmamos que dicha revelación no es sino la continuidad de la manifestación del Poder Creador. Y, finalmente, que esa manifestación constante no se contrapone, sino que presupone una creación inicial con la cual se funde en un proceso creativo complejo. Ahora bien, en concreto, ¿cómo experimenta el existente esta revelación de la divinidad que le permite creer en la creación pasada al experimentar la creación presente? Frente a esta pregunta es menester distinguir necesariamente dos tipos de respuesta, de distinto estatus epistemológico, pero que de ninguna manera son incompatibles. En primer lugar, una respuesta "in theologos" que presupone la revelación positiva e histórica de la divinidad y de su creación, narrada en los textos bíblicos y, por excelencia, en el Génesis. Sobre ello no es posible expedirse "in philosophos". Pero todo hombre en cuanto tal tiene, independientemente de su adhesión a una fe determinada, la posibilidad de experimentar la revelación del Poder Creador. Tal revelación sólo puede ocurrir como la apertura de algo cerrado, como un puro ofrecimiento, como la renovada entrega de sí a través de la cual dicho Poder se manifiesta y llama al existente. Ahora bien: "Es el amor a quien corresponden todas las exigencias que hemos formulado al concepto del Revelador (...)"58. Es, pues, el amor de Dios el modo en que el hombre experimenta la revelación actualísima y acaeciente de su Poder Creador que otorga facticidad a la creación primera. Pero la pregunta se reitera: ¿cómo experimenta el hombre este amor de Dios que actualiza la creación y le permite creer en ella? Desde un punto de vista fenomenológico me parece que una sola respuesta es posible: en cuanto el hombre experimenta, en la más variadas facetas de la existencia, que a él mismo le ha sido dada la capacidad de recrear y reconfigurar el mundo que lo circunda; esto es, en cuanto él experimenta, siendo, que puede ser, que le ha sido dado el poder de ser y que, ejerciéndolo, siendo efectivamente, participa ahora en la obra de la creación. El amor no es una propiedad esencial de Dios, sino el acto renovado a través del cual una y otra vez se patentiza su ser infinito. Se trata de aquel acto por el cual el Poder Creador afirma la existencia del hombre y lo vivifica. Una acción tal se vuelve patente sólo en cuanto el hombre lo experimenta fácticamente, es decir, en cuanto el existente, conmovido por la maravilla de la existencia, afirma la suya y la de todo lo que existe; en otros términos: en cuanto el hombre es y, siendo, vivifica su propia vida y todo aquello que lo rodea. Mas ello precisamente significa, dentro de la lógica de La Estrella, participar en el proceso de consumación de la creación que Rosenzweig llama redención. Por eso mismo la creencia en la creación se manifiesta en la anticipación de una posible redención. Por ello mismo también la creación no se demuestra impugnando absur-

56 Incluso el relato bíblico, como señala S. Mosès, al considerar la creación a lo largo de los días de la semana no la narra como un instante caprichoso, sino como un proceso complejo y sucesivo: "Al fin y al cabo la Biblia no presenta la Creación como el instante en el que el ser hubiera sido arrojado desde la nada, sino como un proceso complejo, distribuido en el tiempo, donde cada día funda su propio orden de realidad: es esta complejidad original lo que garantiza a la ciencia su objetividad." (S. Mosès, "Le dernier journal de Rosenzweig", p. 221).

57 En este sentido escribe Rosenzweig: "Sólo al recibir alguna vez en el tiempo la irradiación del brillo de una revelación no acontecida de una vez y para siempre, sino aconteciendo en este instante, la circunstancia de que las cosas deben su existencia a una revelación llegará a ser más que una circun-stancia; llegará a ser el núcleo íntimo de su facticidad." $(E R, 206)$.

$58 \quad E R, 207$. 
damente teorías científicas verificadas; no se defiende con presuntas pruebas, que en este orden son imposibles y siempre cuestionables, sino que se revela o testimonia cuando el hombre efectiviza el poder creativo que le ha sido dado y, de ese modo, renueva el mundo un día tras otro, aun cuando fuera mínimamente, cuando llena con un poco de sentido cada nueva aurora. Al fin y al cabo, la creación no es un ayer remotísimo disipándose en las brumas de la nada, sino el hecho de poder estar hoy y cada día en camino hacia un nuevo mañana ${ }^{59}$.

59 "Esta providencia de todas las auroras es, pues, a lo que propiamente se alude en el pensamiento de la criatura." $(E R, 165)$. 